\title{
Prematuridade e baixo peso entre recém-nascidos de adolescentes primíparas
}

\author{
Prematurity and low weight of newborns from primiparous adolescents according to age groups
}

Ricardo Cristiano Leal da Rochaㄹ ${ }^{1}$ Eduardo de Souza², Cristina Aparecida Falbo Guazzelli ${ }^{3}$, Antônio Chambô Filho ${ }^{4}$, Eduardo Pereira Soares ${ }^{5}$, Érica da Silva Nogueira ${ }^{5}$

\section{Resumo}

Objetivos: comparar a incidência de partos pré-termos e de recém-nascidos de baixo peso entre adolescentes primíparas de duas faixas etárias. Métodos: estudo clínico, comparativo e transversal, cuja amostra é composta por um universo de 522 adolescentes primíparas, cujos partos ocorreram com idade gestacional entre 25 e 42 semanas. Tais adolescentes foram divididas e avaliadas em dois grupos, de acordo com a faixa etária - Gprec: de 10 a 15 anos completos (n=104); Gtard: de 16 a 19 anos completos $(\mathrm{n}=418)$. Os dados da pesquisa foram obtidos por meio de entrevista individualizada, sigilosa e ética, no puerpério imediato, e mediante questionário escrito, composto por perguntas em relação ao tempo de gestação em semanas completas e ao peso do recém-nascido. A idade gestacional foi calculada no dia do parto, de acordo com a data da última menstruação confiável, sendo ainda confirmada pela ultra-sonografia mais precoce, de até 20 semanas, ou pelo índice de Capurro do recém-nascido, quando na dúvida dos parâmetros anteriormente descritos. Considerou-se prétermo todo recém-nascido com menos de 37 semanas completas de idade gestacional no parto. Consideraram-se de baixo peso ao nascimento todos os recém-nascidos com menos de 2.500 gramas, sendo que o peso dos neonatos foi aferido pelo serviço de neonatologia logo após o parto. Dessa forma, comparamos a taxa de prematuridade e o baixo peso ao nascimento entre recém-nascidos de adolescentes puérperas primíparas. Para a análise estatística, foi empregado o teste $\chi^{2}$ e, para as diferenças localizadas, teste da partição do $\chi^{2}$. Adotou-se o nível de significância de 0,05 (alfa $=5 \%$ ), de forma que níveis descritivos (p) inferiores a esse valor foram considerados significantes. Resultados: a taxa de prematuridade não foi diferente entre os grupos da pesquisa $(5,8$ e 2,6\%). A incidência de baixo peso ao nascimento no Gprec $(13,5 \%)$ foi significativamente maior em relação ao Gtard $(3,1 \%)$. Conclusões: o grupo de adolescentes primíparas na faixa etária mais jovem (inferior a 15 anos) revelou-se de risco significantemente maior para a ocorrência de RN de baixo peso. Não se verificou diferença significante na incidência de partos pré-termos entre os grupos estudados.

PALAVRA-CHAVE: Gravidez na adolescência; Prematuro; Recém-nascido de baixo peso

\section{Abstract}

Purpose: to compare the incidence of preterm deliveries, and of low birth weight newborns, among primiparous adolescents, from two age groups. Methods: this is a comparative, cross-sectional clinical study composed of 522 primiparous adolescents whose deliveries occurred at the gestational age of 25 to 42 weeks. The adolescents were divided into 2 groups according to their age; Gprec: from 10 to 15 complete years old $(n=104)$; Gtard: from 16 to 19 complete years old ( $\mathrm{n}=418)$. The research data were obtained by an individualized, confidential and ethical interview, soon after delivery; and by a written questionnaire with questions about the gestational age in complete weeks, and about the newborns birth weight. The gestational age was calculated at the delivery day, according to the date of the last trustworthy menstrual period, being also confirmed by the earliest pregnancy scanning or by Capurro's index, when there were any doubts about the previously described parameters. All newborns with gestational age under 37 weeks at birth were considered preterm babies. The newborn weight was taken by neonatologists immediately after delivery; all newborns with less than 2,500g were considered to be low weight babies. Thus, we compared prematurity rate and low birth weight among newborns from primiparous puerperal adolescents. The $\chi^{2}$ test was used for the statistic analysis and the partition $\chi^{2}$ test for the found differences.

Serviço de Obstetrícia da Maternidade Pró-Matre de Vitória-ES.

1 Professor Assistente do Departamento de Ginecologia e Obstetrícia da Escola Superior de Ciências da Santa Casa de Misericórdia de Vitória (ES), Brasil.

2 Professor Livre Docente do Departamento de Obstetrícia da Universidade Federal de São Paulo - UNIFESP - São Paulo (SP), Brasil.

3 Professora Doutora do Departamento de Obstetrícia da Universidade Federal de São Paulo UNIFESP - São Paulo (SP), Brasil.

4 Professor Titular do Departamento de Ginecologia e Obstetrícia da Escola Superior de Ciências da Santa Casa de Misericórdia de Vitória (ES), Brasil.

5 Médico do Serviço de Ginecologia e Obstetrícia, Ambulatório de Adolescência, da Santa Casa de Misericórdia de Vitória (ES), Brasil.

Correspondência: Ricardo Cristiano Leal da Rocha

Avenida Nossa Senhora da Penha, 565/1012 - Praia do Canto - 29055-903 - Vitória-ES - e-mail: sogoes@sogoes.com.br 
As the significancy level was $0.05(\alpha=5 \%)$, lower levels than that were considered significant. Results: the prematurity rate was not significantly different between the two groups (5.8 and 2.6\%). The incidence of low birth weight in Gprec $(13.5 \%)$ was significantly higher than in Gtard (3.1\%).Conclusions: the group with primiparous adolescents under 15 years old showed a significantly higher risk of low birth weight newborns. However, a statistically significant incidence of prematurity between the groups studied was not verified.

KEYWORDS: Pregnancy in adolescence; Infant, premature; Low weight newborn

\section{Introdução}

Adolescência é o período compreendido entre a infância e a idade adulta, dos 10 aos 19 anos, caracterizado por intensos crescimento e desenvolvimento, que se manifestam por marcantes transformações anatômicas, fisiológicas, mentais e sociais ${ }^{1,2}$. Na atualidade, a gravidez no extremo inferior da vida reprodutiva, dos 10 aos 19 anos, tem sido objeto de preocupações, pois a gestação, o parto e a maternidade são situações que podem trazer múltiplas conseqüências emocionais, sociais e econômicas para a saúde da mãe adolescente e do seu filho ${ }^{3}$. Com o aumento do número de gestantes adolescentes surgem grandes desafios na perinatologia, em face dos resultados neonatais adversos, encontrados em recém-nascidos de primiparas adolescentes. Na literatura se verifica maior incidência de partos pré-termos e de recémnascidos de baixo peso nesse grupo de gestantes, principalmente entre aquelas com menos de 16 $\operatorname{anos}^{4-7}$. O parto pré-termo e o baixo peso ao nascimento são importantes marcadores de morbidade e mortalidade neonatal e infantil ${ }^{4-7}$.

Tomando como base a estimativa do Instituto Brasileiro de Geografia e Estatística ${ }^{8}$, o Brasil entrou no ano 2000 com 36 milhões de adolescentes. Assim, no nosso pais, emerge o reconhecimento da gravidez na adolescência como um crescente problema de saúde pública ${ }^{7}$.

O parto pré-termo é aquele que ocorre quando a gestação se interrompe antes da $37^{\mathrm{a}}$ semana completa de idade gestacional ${ }^{2}$. A prematuridade, por outro lado, é responsável por cerca de $70 \%$ da taxa de mortalidade perinatal no Brasil ${ }^{9}$.

O baixo peso ao nascer é definido como o peso de nascimento inferior a 2.500 gramas $^{9}$. O parto pré-termo e o baixo peso ao nascimento são eventos que ocorrem com maior freqüência na adolescência, em comparação a mulheres adultas jovens. Além dos riscos biológico, socioeconômico e comportamental nessa faixa etária, a desnutrição, a anemia ferropriva materna, o baixo peso materno ao engravidar, as infecções do trato urinário, as infecções vulvovaginais e a doença hipertensiva específica da gestação são intercorrências que podem contribuir para resultados neonatais adversos ${ }^{10}$.

A incidência de gestação na adolescência no estado do Espírito Santo (21,8\%) é mais alta em relação à média da região sudeste $(19,1 \%)^{8}$. Considerando que esse universo de grávidas tornou-se problema de saúde pública importante no nosso meio em função do aumento dos índices de morbidade e mortalidade neonatal ${ }^{7}$, fomos motivados a estudar o desfecho perinatal entre adolescentes primiparas precoces e tardias.

\section{Métodos}

Foi realizado estudo clínico, comparativo, transversal e prospectivo, com emprego de entrevistas individualizadas e sigilosas. Foram incluídas 522 adolescentes primiparas. As puérperas foram avaliadas por meio de um questionário escrito, padronizado com perguntas dirigidas e objetivas, relacionadas às variáveis em estudo, no puerpério imediato, por um único entrevistador.

As puérperas foram divididas em dois grupos, sendo o Gprec (grupo de adolescentes precoces) na faixa etária de 10 a 15 anos completos e o Gtard (grupo de adolescentes tardias) na faixa etária de 16 a 19 anos completos no dia do parto.

Os dados referentes à gravidez e ao parto foram obtidos por anamnese, cartão de pré-natal, prontuário médico e ficha de identificação do recém-nascido, além de outros exames complementares, observando-se a particularidade de cada caso. Todas as pacientes foram assistidas na maternidade Pró-Matre de Vitória-ES, no período de janeiro de 2000 a dezembro 2002.

A maioria dos recém-nascidos eram filhos de mães adolescentes procedentes de áreas não urbanizadas de Vitória-ES, ou da periferia da região metropolitana. Essas adolescentes apresentaram características socioeconômicas semelhantes, com renda familiar inferior a dois salários minimos. Na maioria das vezes eram solteiras ou viviam em regime de união consensual, sendo em grande parte de baixa escolaridade: haviam cursado apenas o ensino fundamental (Tabela 1). 
A menarca ocorreu, na maioria dos casos, aos treze anos de idade, e grande parte da população nunca havia usado métodos anticoncepcionais. A maior parte das adolescentes não planejou a gestação e tiveram baixa freqüência de consultas no pré-natal, especialmente as adolescentes do Gprec ( Tabela 1).

A idade gestacional variou de 25 a 42 semanas. Para constituir amostra, dentro dos critérios de inclusão, foram consideradas primíparas entre 10 e 19 anos completos e que aceitaram participar do estudo.

O único critério de exclusão foi a não aceitação por parte das adolescentes em participar da entrevista.

O tamanho da amostra foi calculado com base no percentual de incidência de parto pré-termo e baixo peso ao nascimento em conceptos de mães adolescentes, de acordo com a faixa etária, considerando um intervalo de confiança e poder de teste de 95\%, para uma proporção de 1:4. O tamanho ideal da amostra é de 506 pacientes, sendo que o Gprec deveria conter o mínimo de 100 casos, e o Gtard, o mínimo de 400 casos.

Consideramos pré-termos os recém-nascidos com idade gestacional inferior a 37 semanas completas. A idade gestacional foi calculada no dia do parto, de acordo com a data da última menstruação confiável, confirmada pela ultra-sonografia precoce (até 20 semanas) ou pelo índice de Capurro, aplicado quando houve dúvida ou ausência dos parâmetros descritos anteriormente.

Consideraram-se de baixo peso todos os recém-nascidos com menos de 2.500 gramas ao nascimento. O peso foi quantificado em gramas, por meio de balança da marca Filizola, modelo Baby, digital com voltagem para 110 volts, $n^{\circ} 6847 / 00$, série 8201093-6, fabricada em 1996, autorizada e aferida pelo INMETRO. Nesse estudo, o peso dos recém-nascidos foi fornecido pelo serviço de neonatologia da Maternidade Pró-Matre de Vitória-ES,

Tabela 1 - Características da população de adolescentes nos respectivos grupos.

\begin{tabular}{lrrrr}
\hline & \multicolumn{2}{c}{ G (prec) } & \multicolumn{2}{c}{ G (tard) } \\
Característica & $\mathbf{n}$ & $(\%)$ & $\mathbf{n}$ & $(\%)$ \\
\hline Cor da pele não branca & 80 & $(76,9)$ & $280(66,9)$ \\
Habitação em área não urbanizada & 76 & $(73,0)$ & $240(57,4)$ \\
Renda mensal menor ou igual a & 83 & $(79,8)$ & $280(66,9)$ \\
2 salários mínimos & & & \\
Situação conjugal solteira/união consensual & 104 & $(100)$ & $273(65,3)$ \\
Grau de escolaridade: ensino fundamental & 95 & $(91,3)$ & $232(55,5)$ \\
Menarca aos 13 anos & 98 & $(94,2)$ & $325(77,7)$ \\
Não uso de métodos anticoncepcionais & 68 & $(65,3)$ & $214(77,7)$ \\
Gravidez não planejada & 56 & $(65,3)$ & $235(51,2)$ \\
Pré-natal insuficiente <6 consultas & 39 & $(37,5)$ & $81(19,3)$ \\
\hline
\end{tabular}

$G($ prec $)=10$ a 15 anos completos; $G(\operatorname{tard})=16$ a 19 anos completos. logo após o parto. Esta pesquisa foi aprovada pelo Comitê de Ética e Pesquisa da Maternidade Pró-Matre de Vitória-ES.

Para a comparação da incidência de prematuridade e baixo peso ao nascimento entre recém-nascidos de adolescentes primíparas foi aplicado o teste do $\chi^{2}$ e as diferenças localizadas, pelo teste da partição do $\chi^{2}$. Adotou-se o nível de significância de 0,05 (alfa $=5 \%$ ), de forma que níveis descritivos $(\mathrm{p})$ inferiores a esse valor foram considerados significantes.

\section{Resultados}

Durante o período de estudo verificou-se que o percentual de partos entre adolescentes foi de $24 \%$ em relação ao total de partos realizados.

Do total de partos realizados na Maternidade Pró-Matre de Vitória-ES em adolescentes, a incidência de partos no Gprec foi de 19,9\% e no Gtard foi de $81,1 \%$. A idade gestacional em semanas foi apurada como inferior a 37 semanas, de 37 a 42 semanas e acima de 42 semanas. Constatamos, nessa pesquisa, que cerca de $92 \%$ de todas as pacientes tiveram parto entre 37 e 42 semanas de gestação, não havendo diferença entre os dois grupos.

A taxa de pré-termos no grupo Gprec $(n=104)$ foi de $5,8 \%$, ou seja, maior que o dobro do Gtard $(\mathrm{n}=418)$, que correspondeu a 2,6\%. A incidência de baixo peso ao nascimento no Gprec $(n=104)$ teve percentual de $13,5 \%$ e foi significativamente maior em relação ao Gtard $(n=418)$, que correspondeu a $3,1 \%$.

Na Tabela 2, estão nossos resultados obtidos após a análise da incidência de partos pré-termos e do baixo peso ao nascimento entre recém-nascidos de adolescentes primiparas.

$\mathrm{Na}$ análise comparativa entre os grupos estudados (Tabela 2), apuramos que a ocorrência de baixo peso logo após o nascimento no Gprec (13,5\%) foi muito maior em relação ao Gtard $(3,1 \%)$.

Tabela 2 - Distribuição da incidência de parto pré-termo e baixo peso entre recémnascidos de adolescentes primíparas.

\begin{tabular}{|c|c|c|c|c|c|c|}
\hline \multirow{3}{*}{ Característica } & \multirow{2}{*}{\multicolumn{2}{|c|}{$\frac{G \text { (prec) }}{(10 \text { a } 15 \text { anos })}$}} & \multirow{2}{*}{\multicolumn{2}{|c|}{$\frac{G(\text { tard })}{(16 \text { a } 19 \text { anos })}$}} & \multirow{2}{*}{\multicolumn{2}{|c|}{ Total }} \\
\hline & & & & & & \\
\hline & $\mathrm{n}$ & $(\%)$ & $n$ & $(\%)$ & $n$ & $(\%)$ \\
\hline Pré-termo* & 6 & 5,8 & 11 & 2,6 & 17 & 3,3 \\
\hline Baixo peso** & 14 & 13,5 & 13 & 3,1 & 27 & 5,2 \\
\hline Total & 104 & 100 & 418 & 100 & 522 & 100 \\
\hline
\end{tabular}

*Parto que ocorre quando a gestação se interrompe com mais de 22 e menos de 37 semanas completas de idade gestacional; **é definido como peso de nascimento inferior a $2.500 \mathrm{~g}$

$G($ prec $)=10$ a 15 anos completos; $G(\operatorname{tard})=16$ a 19 anos completos

Rev Bras Ginecol Obstet. 2006; 28(9): 530-5. 


\section{Discussão}

O aumento dos índices de gravidez na adolescência pode contribuir para maior prevalência de parto pré-termo e baixo peso ao nascimento ${ }^{4-7}$. A gestação em adolescentes pode ser precoce (até quinze anos) ou tardia (16 aos 19 anos). As grávidas precoces são consideradas de alto risco materno e neonatal ${ }^{10}$.

A etiologia dos aspectos obstétricos da prematuridade é multifatorial. Dentro do universo das causas de parto pré-termo, as condições socioeconômicas adversas, a desnutrição, a anemia ferropriva materna, as infecções vaginais e do trato urinário, a primiparidade jovem e a doença hipertensiva na gravidez são intercorrências que interferem no equilibrio materno-fetal, contribuindo para aumentar os percentuais de partos pré-termo e de recém-nascidos de baixo peso $^{6}$. O parto pré-termo é intercorrência obstétrica com maior freqüência entre adolescentes, em comparação a gestantes de outras faixas etárias ${ }^{4-7}$.

Estudos sobre adolescentes primiparas mostraram percentuais de prevalência de prematuridade variando de 14,3 a $24,3 \%^{11,12}$. Outras pesquisas apontaram incidências de $17 \%$ de parto pré-termo entre puérperas com idade inferior a 16 anos, contra 9\% entre aquelas com idade igual ou superior a $16 \operatorname{anos}^{10}$.

Em um estudo sobre prevalência de parto pré-termo em 1120 adolescentes negras nos Estados Unidos, em relação a mulheres adultas jovens, constatou-se que as menores taxas estão no grupo das adolescentes $(14,5 \%)$, contra $17,4 \%$ no grupo das mulheres adultas jovens ${ }^{13}$.

Já em São Paulo, onde foram avaliadas 499 adolescentes, sendo 160 de 13 a 17 anos e 399 de 18 a 19 anos, verificou-se a incidência de partos pré-termo em 20 e 11,4\%, respectivamente ${ }^{14}$. As adolescentes de menor idade tiveram incidência quase em dobro de partos pré-termo em relação às mais velhas. Investigando 39 adolescentes com menos de 15 anos, 1.393 entre 15 e 19 anos e 2.227 adultas jovens, foram apuradas taxas de parto pré-termo de 7,7, 5,6 e $6 \%$, respectivamente ${ }^{15}$. Nesta amostra, não houve diferença significativa entre os grupos, em que pese ao grupo das adolescentes menores de 15 anos ser composto de amostra pouco expressiva. A análise da prevalência de parto pré-termo em 760 adolescentes e 160 adultas jovens revela percentuais de 14,4 e 2,6\%, respectivamente ${ }^{11}$. Nesse estudo, houve diferença significativa na prevalência de parto pré-termo, sendo que o grupo das adolescentes grávidas precoces teve taxa 4 vezes maior em relação às adultas jovens.

Avaliando a ocorrência de prematuridade em recém-nascidos de 5.687 adolescentes precoces e tardias, e comparando-as com 54.593 adultas jovens, observou-se maior prevalência de parto pré-termo em adultas jovens ${ }^{4}$. Em estudo transversal, utilizando informações do Centro Latino-Americano de Perinatologia, e analisando 854.374 mulheres latino-americanas com menos de 25 anos e 344.626 adolescentes puérperas de gravidez precoce e tardia, foram encontradas incidências de parto pré-termo em 8,9, 14,6 e $10 \%$, respectivamente ${ }^{6}$. Essa incidência foi maior no grupo das adolescentes, principalmente nas precoces, no qual a taxa de nascimento pré-termo foi significativamente maior em relação às adultas jovens.

No nosso estudo, analisamos a taxa de parto pré-termo em adolescentes de gravidez precoce e tardia e verificamos que entre as mais jovens, o nascimento pré-termo teve incidência maior que o dobro em relação às adolescentes grávidas tardias (5,8 contra $2,6 \%)$. No entanto, nossos percentuais foram expressivamente menores em relação aos de outros autores ${ }^{6,13-15}$.

Acreditamos que o tamanho da nossa amostra, menor que aqueles apontados por outros autores já citados, possa justificar os baixos percentuais que encontramos. Outro fator que pode explicar nossos resultados é a adesão pré-natal satisfatória, com 6 ou mais consultas, com percentuais significativos de $62,5 \%$ nas grávidas precoces e $80,7 \%$ nas grávidas tardias. Na maioria dos estudos já citados, os autores referemse a baixa adesão ao pré-natal, chegando inclusive a percentuais da ordem de $23 \%{ }^{16}$. As diferenças geográficas, o nivel de educação materna e as particularidades de cada população podem justificar os resultados dos percentuais diferentes encontrados na literatura.

$\mathrm{Na}$ análise da freqüência de baixo peso ao nascimento, revendo a literatura sobre resultados perinatais de gestação na adolescência, verifica-se que o baixo peso é mais freqüente nos recém-nascidos de adolescentes com faixa etária menor ou igual a $15 \operatorname{anos}^{17}$. Esse grupo etário tem freqüência significativamente maior de recém-nascidos de baixo peso ao nascimento, em relação às adultas jovens. Investigando a prevalência de baixo peso em 1.120 recém-nascidos de mães adolescentes negras nos Estados Unidos e comparando com adultas jovens, verificaram-se percentuais similares (13,3 e 13,5\%). Apurando a incidência de baixo peso em recémnascidos de grávidas precoces, tardias e adultas jovens, outros autores referem-se a porcentagens de $13,2,9,3$ e $8,1 \%$, respectivamente ${ }^{15}$. Nessa pesquisa, a incidência de recém-nascidos de baixo peso foi maior entre as adolescentes, principalmente entre as precoces, em relação às adultas jovens, com diferença significativa.

Em São Paulo, ao avaliar as condições patológicas mais comuns em 152 adolescentes de 13 a 16 anos (29\%) e de 17 a 18 anos $(71 \%)$ os autores referem incidências de baixo peso ao nascimento ${ }^{18}$. No Rio de Janeiro, pesquisadores afirmam que existem efeitos da idade materna na explicação do baixo peso ao nascimento, mesmo quando controlado 
por outras variáveis confundidoras ${ }^{19}$. Estudando resultados perinatais em 6.500 adolescentes de 15 a 19 anos e 6.500 adultas jovens de 20 a 24 anos, encontraram-se percentuais de 9,5 e $6,8 \%$, respectivamente $^{20}$. Taxas maiores e significantes foram encontradas no grupo de menor idade.

Em outro estudo com 941 adolescentes e 3500 adultas jovens, a incidência de baixo peso ao nascimento entre os grupos foi de 17 e 13,5\%, respectivamente ${ }^{20}$. Comparando os dois estudos, verifica-se que a incidência de baixo peso é sempre maior entre recém-nascidos das adolescentes ${ }^{19,20}$.

Na Bahia, após analise de dados do sistema de informação de nascidos vivos referentes a 590 adolescentes de 10 a 16 anos, 1604 de 17 a 19 anos e 3.085 adultas jovens, verificou-se incidência de baixo peso ao nascimento nos grupos de 11,5; 10,5 e $8,9 \%$, respectivamente ${ }^{21}$. O baixo peso ao nascimento foi mais freqüente entre as puérperas adolescentes, principalmente entre as precoces, quando comparadas às adultas jovens, com significância estatística. No Maranhão, ao se investigarem taxas de baixo peso em 319 recém-nascidos de adolescentes menores de 18 anos, 395 de 18 a 19 e 939 de 20 a 24 anos, encontraram-se percentuais de 13,2; 6,3 e 8,2\%, respectivamente ${ }^{22}$. As maiores taxas de recém-nascidos de baixo peso foram entre as grávidas precoces. As grávidas adolescentes tardias tiveram porcentagens de baixo peso menor que as adultas jovens, divergindo assim dos trabalhos já mencionados. Em São Paulo, ao se apurar a incidência de recém-nascidos de baixo peso em 144 adolescentes com média de 16 anos de idade verifica-se taxa de $21 \%{ }^{23}$.

No Rio de Janeiro, o risco gestacional da gravidez entre adolescentes está relacionado aos aspectos clínicos, obstétricos, culturais e socioeconômicos, sendo de natureza multifatorial, e a maior incidência de baixo peso ao nascimento ocorre principalmente entre as grávidas precoces ${ }^{24}$. A adesão tardia ao pré-natal é um dos principais fatores associados ao prognóstico perinatal ${ }^{24}$.

\section{Referências}

1. Organización Panamericana de la Salud. La salud de los adolescentes y los jóvenes en las Américas: escribiendo el futuro. Washington: OPS; 1995. (Comunicación para la Salud, 6).

2. Organización Mundial de La Salud. El embarazo y el aborto en la adolescencia. Ginebra: OMS; 1975. (Série de Informes Técnicos, 583).

3. Coates V, Sant'Anna JC. Gravidez na adolescência. In: Françoso LA, Gejer D, Reato LFN, colaboradores. Sexualidade e saúde reprodutiva na adolescência. São Paulo: Atheneu; 2001. p. 71-84.
Numa análise de 1.800 adolescentes turcas, encontraram-se percentuais de $28,7 \%$ de recémnascidos de baixo peso. Essas taxas são maiores em relação às adultas jovens. A pobreza geral das mães turcas adolescentes e a pouca adesão ao pré-natal (23\%) associadas às diferenças étnicas e socioculturais explicam essas elevadas taxas ${ }^{16}$.

Na verificação da incidência de recém-nascidos de baixo peso em adolescentes grávidas precoces, encontramos percentuais de $13,5 \%$, os quais foram semelhantes aos encontrados por outros autores ${ }^{13,15,24}$. Apuramos menores taxas de recém-nascidos de baixo peso em relação aos achados de outros autores ${ }^{23}$. Ainda, no nosso estudo, encontramos maiores porcentagens de nascimento de baixo peso em relação aos estudos realizados por outros pesquisadores ${ }^{18,21}$.

Quanto à incidência de recém-nascido de baixo peso entre adolescentes grávidas tardias, apuramos taxas de $3,1 \%$. Os nossos resultados revelam menor incidência de baixo peso ao nascimento em relação aos trabalhos publicados por outros autores ${ }^{15,16,18-24}$.

Acreditamos que as diferenças étnicas, socioculturais, localização geográfica, risco social (baixo nivel de escolaridade, baixa adesão ao pré-natal, renda mensal de 2 ou menos salários mínimos, peso materno abaixo do ideal e hábitos alimentares inadequados antes e durante a gestação) possam ter interferido na incidência de baixo peso ao nascimento. É importante lembrar que outras limitações metodológicas como amostras pequenas, falta de especificidade para testar associações, falta de definições uniformes e de controle de fatores confundidores possam justificar os diferentes percentuais encontrados na literatura.

Concluímos que a gestação na adolescência é fenômeno em ascensão em nosso meio que envolve aspectos relacionados à saúde pública. As complicações perinatais como o nascimento pré-termo e o baixo peso ao nascer dependem não somente da idade da gestante (risco biológico), mas principalmente das condições de riscos social e econômico.

4. Pardo RA, Nazer J, Cifuentes L. Prevalencia al nacimiento de malformaciones congénitas y de menor peso de nacimiento en hijos de madres adolescentes. Rev Med Chile. 2003;131(10):1165-72.

5. Wallace JM, Aitken RP, Milne JS, Hay WW Jr. Nutritionally mediated placental growth restriction in the growing adolescent: consequences for the fetus. Biol Reprod. 2004;71(4):1055-62.

6. Conde-Agudelo A, Belizán JM, Lammers C. Maternalperinatal morbidity and mortality associated with adolescent pregnancy in Latin America: cross-sectional study. Am J Obstet Gynecol. 2005;192(2):342-9. 
7. Goldenberg P, Figueiredo MCT, Silva RS. Gravidez na adolescência, pré-natal e resultados perinatais em Montes Claros, Minas Gerais, Brasil. Cad Saúde Pública. 2005;21(4):1077-86.

8. Instituto Brasileiro de Geografia e Estatística [homepage na Internet]. Censo Demográfico 2000: nupcialidade e fecundidade: resultados da amostra. Rio de Janeiro: IBGE; 2003. [citado 2005 Out 19]. Disponivel em: http:/ /www. ibge.gov.br/home/estatistica/ populacao/censo2000/ nupcialidade_fecundidade/censo2000_fecundidade.pdf

9. World Health Organization: recommended definitions, terminology and format for statistical tables related to the perinatal period and use of a new certificate for cause of perinatal deaths. Modifications recommended by FIGO as amended October 14, 1976. Acta Obstet Gynecol Scand. 1977;56(3):247-53.

10.Maia Filho NL, Tedesco RP, Neder VM. Comparação entre os resultados obstétricos de adolescente precoces e tardias após três décadas de prevenção. GO Atual. 1999;3(1/2):14-22.

11.Ziadeh S. Obstetric outcome of teenage pregnancies in North Jordan. Arch Gynecol Obstet. 2001;265(1):26-9.

12. Grady MA, Bloom KC. Pregnancy outcomes of adolescents enrolled in a Centering Pregnancy program. J Midwifery Womens Health. 2004;49(5):412-20.

13. Chang SC, O’Brien KO, Nathanson MS, Mancini J, Witter FR. Characteristics and risk factors for adverse birth outcomes in pregnant black adolescents. J Pediatr. 2003;143(2):250-7.

14.Ribeiro ERO, Barbieri MA, Bettiol H, Silva AAM. Comparação entre duas coortes de mães adolescentes em município do Sudeste do Brasil. Rev Saúde Pública. 2000;34(2):136-42.

15.Gallo PR, Reis AOA, Leone C. Características de seguimento pré-natal, do parto e do recém-nascido de adolescentes grávidas, município de Joinville, Estado de Santa Catarina, Brasil, 1995. Pediatria (São Paulo). 2000;22(2):123-9.
16. Yldirim Y, Inal MM, Tinar S. Reproductive and obstetric characteristics of adolescent pregnancies in Turkish women. J Pediatr Adolesc Gynecol.2005;18(4): 249-53.

17.Kurauchi ATN, Roteli-Martins CM, Aquino MMA. Impacto da gravidez na adolescência e resultados perinatais: revisão de literatura. Femina. 2003;31(8):669-71.

18. Dotta IG, Noda E, Silva SLL. Gestação na adolescência. RBM Cad Ginecol Obstet. 2000;57(1): 17-22.

19. Gama SGN, Szwarcwald CL, Leal MC, Theme Filha MM. Gravidez na adolescência como fator de risco para baixo peso ao nascer no município do Rio de Janeiro, 1996 a 1998. Rev Saúde Pública. 2001;35(1):74-80.

20.Gama SGN, Szwarcwald CL, Leal MC. Experiência de gravidez na adolescência, fatores associados e resultados perinatais entre puérperas de baixa renda. Cad Saúde Pública. 2002;18(1):153-61.

21.Costa MC, Santos CAT, Sobrinho CLN, Freitas JO, Ferreira KASL, Silva MA, et al. Estudo dos partos e nascidos vivos de mães adolescentes e adultas jovens no Município de Feira de Santana, Bahia, Brasil, 1998. Cad Saúde Pública. 2002;18(3): 715-22.

22.Simões VMF, Silva AAM, Bettiol H, Lamy-Filho F, Tonial SR, Mochel EG. Características da gravidez na adolescência em São Luís, Maranhão. Rev Saúde Pública. 2003;37(5):559-65.

23. Basílio RL, Bedin MR, Oliveira MCC. Perfil das mães adolescentes que deram à luz no Hospital Materno-Infantil da Faculdade de Medicina de Marília entre outubro de 1999 e setembro de 2000 e o produto de tais concepções. GO Atual. 2003;3(8/9):12-6.

24.Bouzas I, Miranda AT. Gravidez na adolescência. Rev Adolesc Saúde. 2004;1(1):27-30. 